body $^{3}$ and hence likely to also be transported to the central terminal. This mirror image distribution is paralleled by numerous proteins including the vesicularly contained peptides substance $\mathrm{P}$ and CGRP, opioid and vanilloid receptors and some enzymes ${ }^{4}$. Thus, the presence of PAR2s on the central terminals of axons (Fig. 1) would provide an important communication system linking the local milieu-this time in the dorsal horn-to the central PAR2-bearing afferent terminal.

It is also interesting to note that the present study reports that the hyperalgesia induced by the activation of peripheral PAR2s was reversed by the systemic, but not local, application of the COX inhibitor indomethacin, indicating a central action ${ }^{5}$. Centrally mediated release of a large enzyme such as trypsin is not without precedence. The enzyme PLA2 is released and diffuses to other cells where it is believed to be internalized and involved in the generation of arachidonic acid, a necessary substrate for prostaglandin formation ${ }^{6}$.

Interestingly, a variety of cell systems including microglia and astrocytes express proteinases when activated. Although it is generally believed that this expression occurs in the face of local destruction-as with spinal injury, ${ }^{7,8}$-there is no current reason to exclude the possibility that this signaling system is brought into play by relatively common events that activate these non-neuronal spinal systems. In a variety of chronic pain conditions there is prominent proliferation and activation of astrocytes and/or microglia ${ }^{9-11}$. Circulating cytokines believed to contribute to systemic pain states ${ }^{12}$ will activate populations of perivascular microglia, endothelial cells and leptomeninges within the CNS, leading to increased cyclo-oxyegenase (COX) expression $^{13-15}$. These activated cells could also generate increases in extracellular proteinases.

The work of Vergnolle et al. on peripheral PAR2 provides novel insights that not only add to our growing understanding of the biology of the primary afferent, but also suggests additional pharmacological targets for analgesics that act on the primary afferent axon. If present speculation regarding the potential role of PARs within the spinal cord and the release of proteinases from activated spinal cells bears out, the spinal dorsal horn would prove to be an additional therapeutic target.

1. Vergnolle, N. et al. Proteinase-activated receptor-2 and hyperalgesia: A novel pain pathway. Nature Med. 7, 821-826 (2001).

2. O’Brien, P.J., Molino, M., Kahn, M. \& Brass, L.F. Protease activated receptors: theme and variations Oncogene 20, 1570-1581 (2001).

3. Steinhoff, M. et al. Agonists of proteinase-activated receptor 2 induce inflammation by a neurogenic mechanism. Nature Med. 6, 151-158 (2000).

4. Hunt, S.P. \& Mantyh, P.W. The molecular dynamics of pain control. Nat. Rev. Neurosci. 2, 83-91 (2001).

5. Malmberg, A.B. \& Yaksh, T.L. Hyperalgesia mediated by spinal glutamate or substance $\mathrm{P}$ receptor blocked by spinal cyclooxygenase inhibition. Science 257, 1276-1279 (1992).

6. Balsinde, J., Balboa, M.A., Insel, P.A. \& Dennis, E.A. Regulation and inhibition of phospholipase A2. Annu. Rev. Pharmacol. Toxicol. 39, 175-189 (1999).
7. Ray, S.K. et al. Calpain activity and translational expression increased in spinal cord injury. Brain Res. 816, 375-380 (1999).

8. Shields, D.C., Schaecher, K.E., Hogan, E.L. \& Banik, N.L. Calpain activity and expression increased in activated glial and inflammatory cells in penumbra of spinal cord injury lesion. J. Neurosci. Res. 61, 146-150 (2000)

9. DeLeo, I.A. \& Yezierski, R.P. The role of neuroinflammation and neuroimmune activation in persistent pain. Pain 90, 1-6 (2001).

10. Fu, K.Y., Light, A.R. \& Maixner, W. Relationship between nociceptor activity, peripheral edema, spinal microglial activation and long-term hyperalgesia induced by formalin. Neuroscience 101, 1127-1135 (2000).

11. Honore, P. et al. Murine models of inflammatory, neuropathic and cancer pain each generate a unique set of neurochemical changes in the spinal cord and sensory neurons. Neuroscience $98,585-598$ (2000).

12. Watkins, L.R. \& Maier, S.F. Implications of immune-tobrain communication for sickness and pain. Proc. Natl. Acad. Sci. USA 96, 7710-7713 (1999).

13. Quan, N., Whiteside, M. \& Herkenham, M. Cyclooxygenase 2 mRNA expression in rat brain after peripheral injection of lipopolysaccharide. Brain Res. 802, 189-197 (1998).

14. Matsumura, K. et al. Brain endothelial cells express cyclooxygenase-2 during lipopolysaccharide-induced fever: Light and electron microscopic immunocytochemical studies. I. Neurosci. 18 6279-6289 (1998)

15. Lacroix, S. \& Rivest, S. Effect of acute systemic inflammatory response and cytokines on the transcription of the genes encoding cyclooxygenase enzymes (COX-1 and (COX-2) in the rat brain. J. Neurochem. 70, 452-466 (1998).

${ }^{1}$ Department of Preventive Science, Neuroscience and Psychiatry

University of Minnesota

Minneapolis, Minnesota, USA

Email:manty001@tc.umn.edu

${ }^{2}$ Department of Anesthesiology

University of California, San Diego

San Diego, California, USA

Email:tyaksh@ucsd.edu

\title{
Heart might repair itself
}

A report in the June 7 issue of the New England Journal of Medicine provides striking evidence that heart cells regenerate after a heart attack. Anversa and colleagues at the New York Medical College in Valhalla, New York found large-scale replication of heart muscle cells, as well as other indicators of cell regeneration, in zones of the heart bordering the infarct area.

A number of studies in recent years, including one from the same authors, have challenged the notion that damage to the heart after a heart attack is permanent. The new findings, however, provide the most convincing evidence so far.

Using confocal microscopy, Anversa's group compared myocytes from the hearts of 13 patients, 4 to 12 days after they suffered heart attacks, with myocytes from patients who had died of other causes. They found that in heart attack patients, the number of myocytes positive for the Ki-67 antigen, a marker of cell division, was 78 times higher in tissue samples from the border zone of the infarct and 28 times higher in samples from the distant myocardium (compared with controls). Further evidence that myocytes regenerated was obtained through images of nuclear mitotic division and cytokinesis. The latter event, depicted in the photograph, was identified by labeling nuclei with propidium iodide (green fluorescence) and staining myocyte cytoplasm with an antibody against sarcomeric $\alpha$ actin (red fluorescence).

The next step in this work will be to iden-

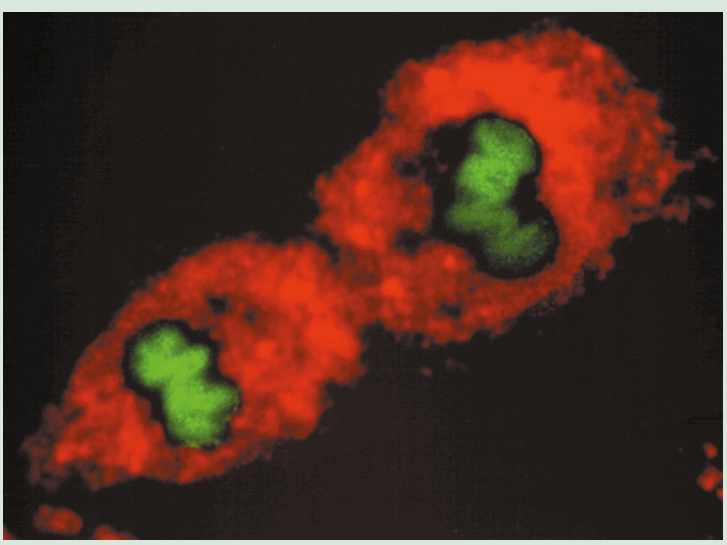

tify the source of the dividing myocytes. It is possible that the dividing myocytes represent a subpopulation of known cells that retain the capacity to divide or cells that originate from yet unidentified stem cells in the human heart.

LAURA BONETTA 